\title{
Graphene-doped polymer nanofibers for low-threshold nonlinear optical waveguiding
}

\author{
Chao Meng ${ }^{1 *}$, Shao-Liang Yu ${ }^{1 *}$, Hong-Qing Wang ${ }^{2 *}$, Yue Cao $^{2}$, Li-Min Tong $^{1}$, Wei-Tao Liu ${ }^{2}$ and Yuen-Ron Shen ${ }^{3}$ \\ Graphene-doped polymer nanofibers are fabricated by taper drawing of solvated polyvinyl alcohol doped with liquid-phase exfoliated \\ graphene flakes. Nanofibers drawn this way typically have diameters measured in hundreds of nanometers and lengths in tens of \\ millimeters; they show excellent uniformity and surface smoothness for optical waveguiding. Owing to their tightly confined \\ waveguiding behavior, light-matter interaction in these subwavelength-diameter nanofibers is significantly enhanced. Using \\ approximately $1350-\mathrm{nm}$-wavelength femto-second pulses, we demonstrate saturable absorption behavior in these nanofibers with a \\ saturation threshold down to $0.25 \mathrm{pJ}$ pulse ${ }^{-1}$ (peak power $\sim 1.3 \mathrm{~W}$ ). Additionally, using 1064-nm-wavelength nanosecond pulses as \\ switching light, we show all-optical modulation of a $1550-\mathrm{nm}$-wavelength signal light guided along a single nanofiber with a switching \\ peak power of $\sim 3.2 \mathrm{~W}$.
}

Light: Science \& Applications (2015) 4, e348; doi:10.1038/Isa.2015.121; published online 6 November 2015

Keywords: graphene; nanofiber; optical modulation; saturable absorption

\section{INTRODUCTION}

Graphene and its derivatives have attracted considerable interest as materials for micro/nano-photonic devices because of their exceptional optical and electronic properties, which include a gapless band structure $^{1}$, universal ultra-broadband optical absorption ${ }^{2,3}$, controllable carrier doping ${ }^{4,5}$, ultrahigh charge-carrier mobility ${ }^{6}$, and ultrafast carrier relaxation time $e^{7-9}$. However, single-layer graphene absorbs only about $2 \%$ of transmitted light, which is too low for many photonic functionalities. The integration of graphene with photonic micro/nanostructures (e.g., optical waveguides and microcavities $^{10-19}$ ) can strongly enhance the graphene-light interaction, and has been used in many applications ranging from photodetectors $^{12,14,15}$ to optical polarizers ${ }^{11}$, ultrafast lasers ${ }^{10}$, and optical modulators $^{13,16,17}$. Optical waveguides are particularly attractive due to their significantly enhanced interaction length, compact size, and high integrability, but hybridizing graphene film with a micro/nanowaveguide involves elaborate and complicated transferring processes $^{13,19}$ that are time-consuming and difficult to adapt to waveguides on a subwavelength scale. Furthermore, although field intensity inside the waveguide is much stronger than outside ${ }^{20}$, graphene film usually has to be pasted outside the waveguide ${ }^{13,17,19}$. Recently, polymer optical nanofibers have been demonstrated to be excellent hosts for a number of functional dopants such as dye molecules $^{21}$, quantum dots ${ }^{22}$, and gold nanorods ${ }^{23}$, and have shown great promise in nanophotonics with great versatility and high compactness ${ }^{24-28}$. Compared to most other functional dopants (e.g., quantum dots or dyes), graphene has many outstanding properties including no photo-bleaching, much higher optical nonlinearity and ultra-broadband compatibility.

Here, we report on the successful fabrication of graphene-doped polymer nanofibers, drawn from a solvated polymer with incorporated liquid-phase exfoliated (LPE) graphene flakes, which exhibit excellent optical and mechanical properties ${ }^{29}$. With a controllable amount of dopant, these nanofibers may exhibit broadband ultrafast saturable absorption with very low pulse energy, suggesting they have possible applications in ultra-low-threshold saturable absorption and all-optical modulation. Using nanosecond pulses as pump light, we show all-optical modulation of the transmission of continuum-wave (CW) light through a single nanofiber.

\section{MATERIALS AND METHODS}

Preparation of graphene flakes

Graphite powders (1.1 wt-\%) were exfoliated into single- and fewlayer graphene sheets in water using sodium deoxycholate (SDC) (0.44 wt-\%) as a bile salt surfactant through tip sonication (BRASON S450A; Emerson Electric Co., Danbury, CT, USA) ${ }^{29}$. To remove thick graphite materials (such as un-exfoliated graphite and large graphite particles) from the water, the prepared dispersion was left standing overnight. The top $80 \%$ of the graphene dispersion was decanted and centrifuged at $12000 \mathrm{r} \mathrm{min}^{-1}$ for one hour, leaving a gray supernatant that predominantly contained single-layer and few-layer graphene, as shown in the first step of Figure 1. The lateral dimensions of the obtained graphene flakes ranged from a few hundred nanometers to several micrometers (Supplementary Information Fig. S1). The con-

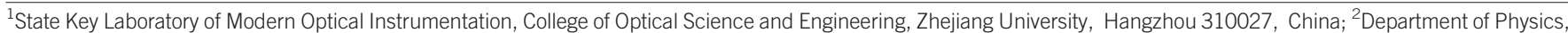

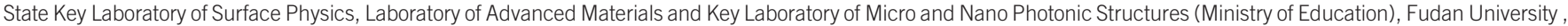
Shanghai 200433, China and ${ }^{3}$ Department of Physics, University of California, Berkeley, CA 94720, USA.

*These authors contributed equally to this work.

Correspondence: LM Tong, Email:phytong@zju.edu.cn; WT Liu, Email: wtliu@fudan.edu.cn

Received 12 February 2015; revised 21 June 2015; accepted 22 June 2015; accepted article preview online 29 June 2015 
centration of graphene in the aqueous suspension, as derived from the absorption spectrum (Supplementary Information Fig. S2), was approximately $0.2 \mathrm{mg} \mathrm{mL}^{-130}$.

\section{Preparation of graphene-doped polymer nanofibers}

Optical-quality graphene-doped PVA (G/PVA) nanofibers were fabricated using a direct drawing method. To prepare graphene-doped polymer, $8 \mathrm{~mL}$ of graphene dispersion were doped with $200 \mathrm{mg}$ polyvinyl alcohol (PVA), followed by magnetic stirring for 4 hours at $80{ }^{\circ} \mathrm{C}$ to obtain an optically homogeneous G/PVA solution, as shown in Figure 1c. PVA was chosen as the host polymer because of its high transparency in the visible and near-infrared region, excellent solvent compatibility with graphene flakes, and good mechanical and thermal properties $^{31,32}$. A tungsten tip was then used to draw nanofibers from the doped polymer, as shown in Figure 1d.

\section{RESULTS AND DISCUSSION}

The drawn G/PVA nanofibers were typically hundreds of nanometers in diameter and tens of millimeters in length. Their diameters were uniform along their lengths and their surfaces were smooth (Figure 2a). The dopant did not appreciably change the structural and mechanical properties of the nanofiber. Such nanofibers can be readily incorporated as integrated components, such as microcavities or junctions, into a photonic circuit. For example, by micromanipulation under an optical microscope ${ }^{24}$, a G/PVA nanofiber can be assembled into a loop (Figure 2b, also a knot in Supplementary Information Fig. S3) or a spiral (Figure 2c) without failure.

We used transmission electron microscopy (TEM) and micro Raman spectroscopy to characterize the G/PVA nanofibers. The TEM image (Figure 2d) of a G/PVA nanofiber shows that graphene flakes (appearing as small dark patches) were spread out in the fiber. Figure 2e compares the Raman spectra of a G/PVA nanofiber, a pure

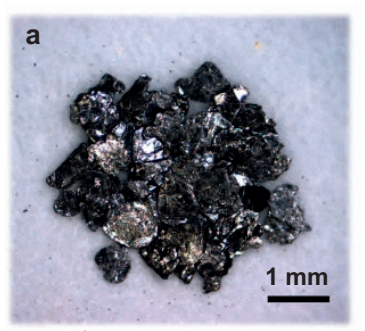

Graphite powders
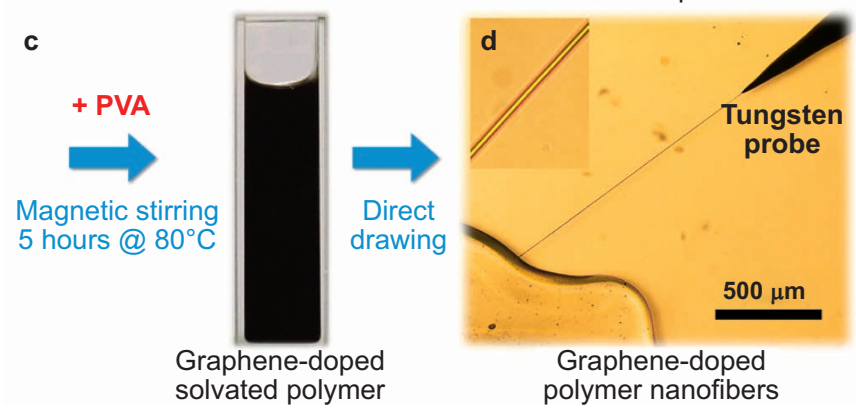

Figure 1 Schematic illustration of the fabrication procedure for creating G/PVA nanofibers. (a, b) Graphite powder was exfoliated into an aqueous graphene dispersion using sodium deoxycholate (SDC) as a bile salt surfactant. (c) Doped with polyvinyl alcohol (PVA), the graphene aqueous solution was magnetically stirred to create solvated G/PVA. (d) G/PVA nanofibers were directly drawn from the solvated G/PVA using a tungsten probe.
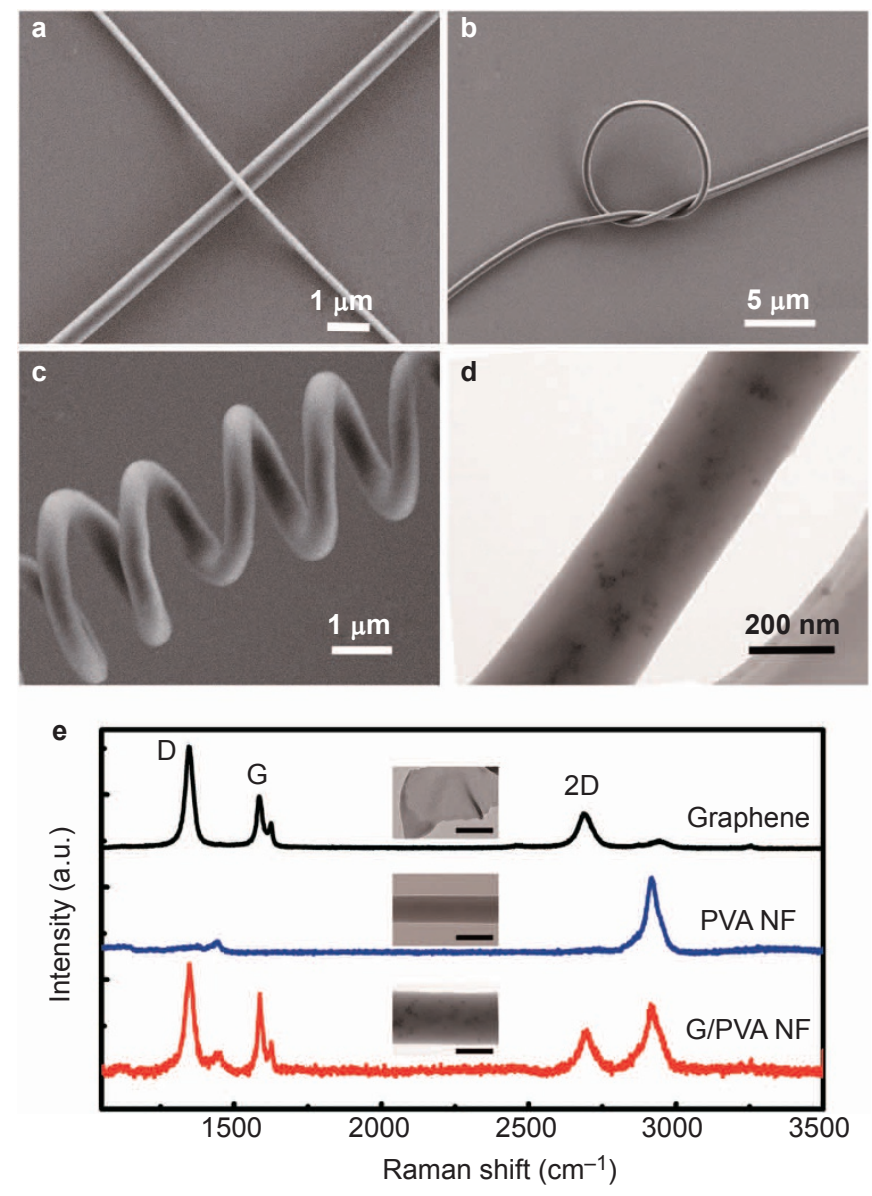

Figure 2 Electron microscopy and Raman characterization of G/PVA nanofibers. (a-c) SEM images of (a) 170- and 510-nm-diameter G/PVA nanofibers, (b) a 7- $\mu$ m-diameter micro-knot tied with a 600-nm-diameter G/PVA nanofiber and (c) a micro spiral assembled with a 480-nm-diameter G/PVA nanofiber. (d) TEM image of a 290-nm-diameter G/PVA nanofiber. (e) Raman spectra of a LPE graphene flake (upper), a pure PVA nanofiber (middle), and a G/PVA nanofiber (bottom). The insets show TEM images of the corresponding samples. Scale bars, $200 \mathrm{~nm}$.

PVA nanofiber, and an LPE graphene flake on a silicon substrate. The $\mathrm{G}, \mathrm{D}$, and $2 \mathrm{D}$ modes of graphene are clearly visible in the spectrum of G/PVA, indicating the presence of graphene in the fiber. The $2 \mathrm{D}$ peak appearing as a single Lorentzian peak is characteristic of monolayer graphene $^{32,33}$.

To study linear transmission of the G/PVA nanofibers, incoherent white light (a fiber-coupled halogen lamp, spectrum given in Supplementary Information Fig. S4) was coupled to the G/PVA nanofiber at one end via the fiber-taper-assistant evanescent coupling scheme $^{24}$ (Supplementary Information), and transmitted light was detected at the other end. Both the G/PVA nanofiber and the fiber taper were freestanding in air to eliminate the short-pass filtering effect. Figure 3a shows an optical microscope image of an 800-nmdiameter G/PVA nanofiber guiding green and red lights. Compared with the relatively strong output (right-hand output spot of the nanofiber), sidewall scattering is negligible (Supplementary Information Fig. S5), indicating no obvious aggregation of graphene flakes in the fiber (Figure $2 \mathrm{~d}$ ). Figure $3 \mathrm{~b}$ shows a normalized broadband transmittance of a typical 100- $\mu \mathrm{m}$-long, 720-nm-diameter G/PVA nanofiber. Transmittance at the long-wavelength side $(>1200 \mathrm{~nm})$ seems higher 

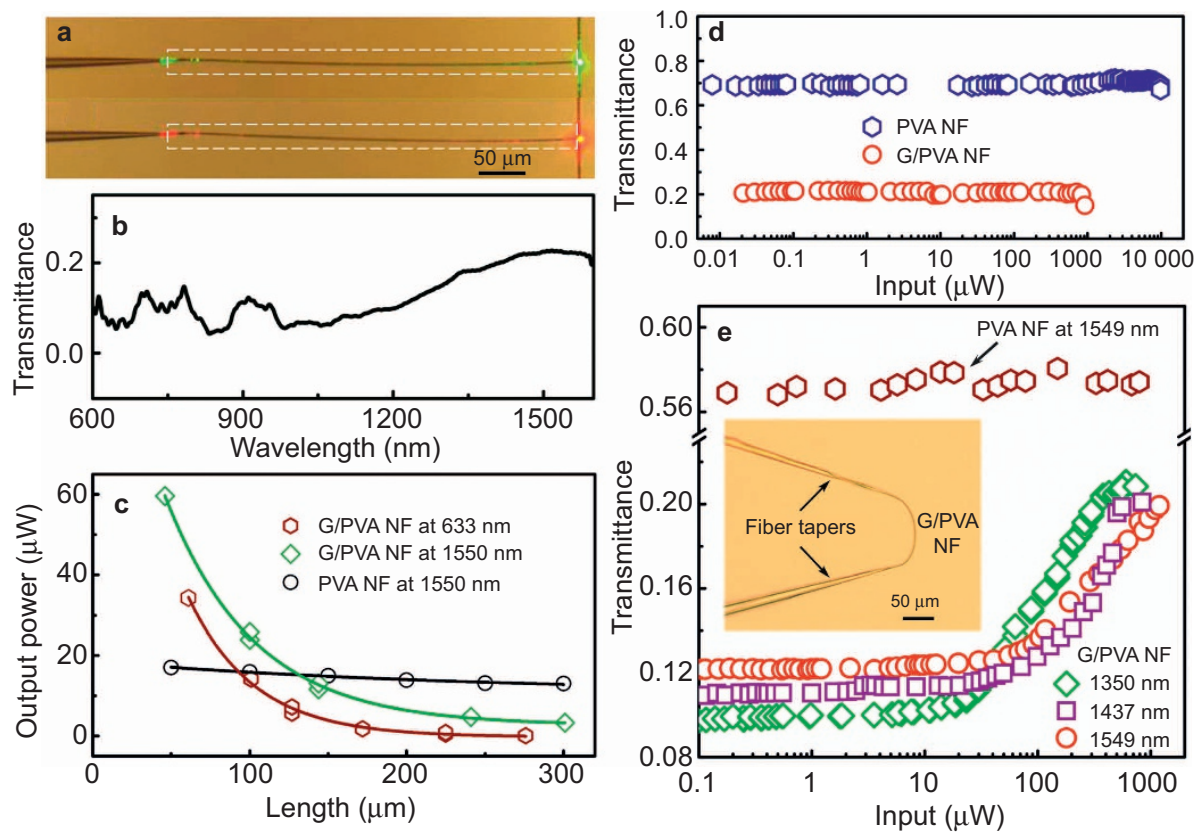

Figure 3 Optical characterization of G/PVA nanofibers. (a) Optical microscopy image of an 800-nm-diameter G/PVA nanofiber waveguiding green (top) and red (bottom) light. The G/PVA nanofiber is marked with dashed boxes. (b) Typical broadband transmittance of a 100- $\mu$ m-long, 720-nm-diameter G/PVA nanofiber. (c) Propagation-distance-dependent output optical intensities of a G/PVA nanofiber at 633- and 1550-nm wavelengths. (d) Optical damage threshold measurements of a pure PVA nanofiber (blue hexagons) and a G/PVA nanofiber (red circles) waveguiding 1550-nm-wavelength continuous-wave light. (e) Broadband saturable absorption behaviors of single G/PVA nanofibers guiding femtosecond pulses and power-dependent transmittance of a pure PVA nanofiber. Inset is an optical microscope image of a 100- $\mu \mathrm{m}$-long, 800-nm-diameter G/PVA nanofiber coupled to two fiber tapers in air. To facilitate in/out light coupling, the nanofiber is coupled to two fiber tapers.

than the rest, which can be explained by lower fractional power confined in the fiber at longer wavelength ${ }^{20}$.

We quantitatively measured the linear absorption coefficient of a representative G/PVA nanofiber $(650 \mathrm{~nm}$ in diameter and doped with $\sim 0.8$ wt- $\%$ of graphene flakes) at selected wavelengths using CW lasers. The laser light was coupled to the nanofiber at different positions to vary propagation length in the nanofiber, and the absorption coefficient was deduced from the exponential decay of the transmitted light and increase in propagation length. The results displayed in Figure $3 \mathrm{c}$ yield absorption coefficients of $0.11 \mathrm{~dB} \mu \mathrm{m}^{-1}(0.024$ $\left.\mu \mathrm{m}^{-1}\right)$ at $633-\mathrm{nm}$ wavelength and $0.069 \mathrm{~dB}_{\mu \mathrm{m}^{-1}}\left(0.016 \mu \mathrm{m}^{-1}\right)$ at a 1550-nm wavelength of a G/PVA nanofiber, which are lower than many other functionally doped polymer nanofibers ${ }^{34,35}$; this is due to the excellent surface quality and relatively low concentration of graphene dopants. For reference, the length-dependent output of a pure PVA nanofiber is also provided, with an absorption coefficient of approximately $0.004 \mathrm{~dB}_{\mu \mathrm{m}^{-1}}\left(0.0001 \mu \mathrm{m}^{-1}\right)$ at a $1550-\mathrm{nm}$ wavelength. The excellent exponential fitting of the propagation length dependent output power of the G/PVA nanofiber shows that, on average, the graphene has been uniformly doped along the length of the nanofiber. By changing the concentration of graphene flakes in the solvated polymers before fiber drawing (Supplementary Information Fig. S2b), the absorption coefficient of the G/PVA nanofibers can be changed. Because graphene absorption converts guided light into heat, the optical damage threshold of the G/PVA nanofiber is lower than that of the pure PVA nanofiber. As shown in Figure 3d, the abrupt drop in transmittance of continuous wave 1550-nm light at high power marks a damage threshold of approximately $10 \mathrm{~mW}$ for the pure PVA nanofiber (blue hexagons) and $1 \mathrm{~mW}$ for the G/PVA nanofiber (red circles).
With pulsed laser excitation, the band filling (Pauli blocking) effect of the exited carriers can drastically change the absorption spectrum of graphene and lead to saturable absorption of the G/ PVA nanofibers ${ }^{31,32,36,37}$. To investigate this effect, we used fiber tapers

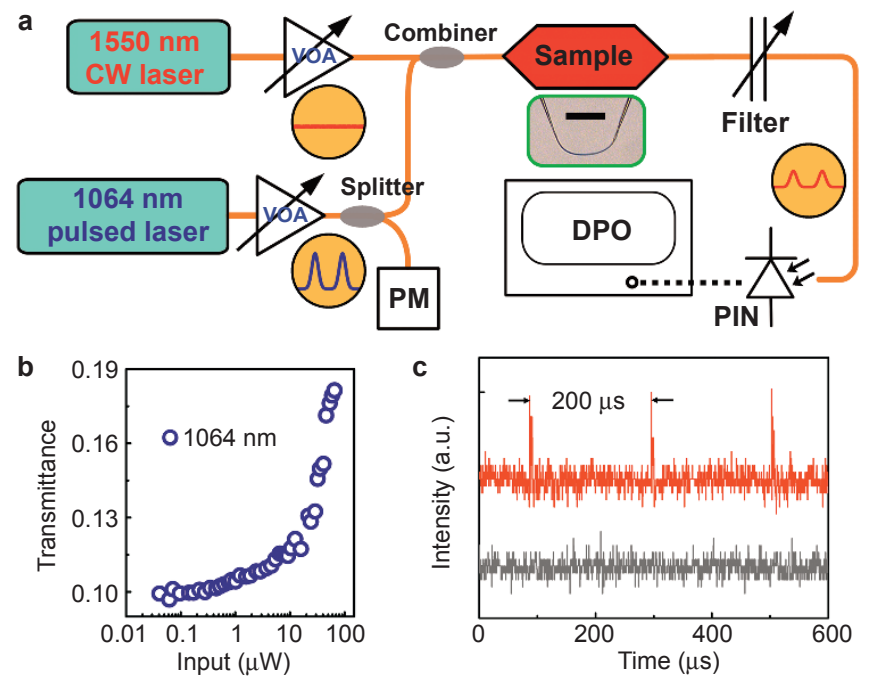

Figure 4 G/PVA nanofiber for optical modulation. (a) Schematic illustration of the experimental setup. The inset shows a microscope image of one G/PVA nanofiber coupling with two fiber tapers in air. Scale bars, $50 \mu \mathrm{m}$. (b) Inputpower-dependent nonlinear transmittance of a 120- $\mu$ m-long G/PVA nanofiber guiding 1064-nm-wavelength nanosecond pulses. (c) Optical modulation of a CW 1550-nm-wavelength light by 1064-nm-wavelength nanosecond pulses (average power of $\sim 80 \mu \mathrm{W}$ ) in a single G/PVA nanofiber. The gray line represents the measured signal output with the switch light on and the signal light off. 
at both ends of a 800-nm-diameter, 100- $\mu \mathrm{W}$-long, G/PVA nanofiber to couple light in and out of the fiber (inset of Figure 3e), and used low-average-power ultrafast pulses ( $\sim 200 \mathrm{fs}, 78 \mathrm{MHz}$ ) from an optical parametric oscillator $(\mathrm{OPO})$ as a probe. The two fiber tapers were angularly separated to avoid direct communication between the two. Figure 3e plots the power-dependent transmittance of the nanofiber at 1350-, 1437-, and 1549-nm wavelengths. At an average power below $10 \mu \mathrm{W}$, the nanofiber shows linear transmittance of approximately $10 \%$ at all three wavelengths; when the power was increased to $\sim 10-20 \mu \mathrm{W}$, transmittance started to increase with increasing power due to saturated absorption. Because the optical field is better confined in a nanofiber at shorter wavelength ${ }^{20}$, the linear transmittance and saturable absorption threshold of the G/PVA nanofiber decreases as wavelength decreases. At $1350 \mathrm{~nm}$, the saturable absorption threshold is $\sim 20 \mu \mathrm{W}$, corresponding to a pulse energy of $\sim 0.25 \mathrm{pJ}$ pulse $^{-1}$ ( $\sim 1.3 \mathrm{~W}$ peak power). At power of $\sim 200 \mu \mathrm{W}$, the transmittance changes from $10 \%$ to $21 \%$, which is a larger change than those in previously reported composite graphene/PVA films (e.g., from $64.4 \%$ to $65.7 \%$ [Ref. 32], and from $26.5 \%$ to $28.6 \%$ [Ref. 38]). Higher graphene doping concentration can lead to larger power-dependent changes while still maintaining reasonable linear transmittance ${ }^{36}$. For reference, the power-dependent transmittance of a pure PVA nanofiber is also presented in Figure $3 \mathrm{~d}$, showing no evident saturated absorption when the graphene dopants are absent.

Saturable absorption can be utilized for optical modulation or switching of light. Here, we demonstrate the use of a G/PVA nanofiber for all-optical modulation at approximately 1550-nm wavelength (the C-band of optical communication) with low power consumption and a miniaturized footprint. As schematically illustrated in Figure 4a, the measurement was arranged using fiber tapers at both ends of a G/PVA nanofiber to couple light in and out. In turn, the fiber tapers were connected to standard fiber circuits for the alloptical modulation test. In the experiment, the signal beam (1550-nm CW light) and modulating beam (1064-nm, 5-ns laser pulses with a repetition rate of $5 \mathrm{kHz}$ ) were coupled to standard optical fibers, combined with co-propagation beams and sent into the nanofiber via a fiber taper. Limited by the damage threshold of the G/PVA nanofiber ( $\sim 1 \mathrm{~mW}$ for $\mathrm{CW}$ light), the CW signal light was kept below $0.5 \mathrm{~mW}$. Additionally, to facilitate the detection of transient modulation on the low-power CW light, here we used nanosecond (instead of femto- or pico-second) pulses as switching light. The nanofiber was $120-\mu \mathrm{m}$ in length, $800-\mathrm{nm}$ in diameter, and doped with $\sim 0.8 \mathrm{wt}-\%$ graphene. The signal output was collected by the fiber taper at the output end, filtered by an in-line fiber-optic notch filter to eliminate the modulating pulses, and finally directed to a photodetector (PIN). The power of the modulating pulses was monitored by an optical power meter (PM). We first measured the power-dependent transmittance of the modulating beam without the in-line notch filter in place. The result, shown in Figure $4 \mathrm{~b}$, was a threshold of saturable absorption at $\sim 10 \mu \mathrm{W}(\sim 0.4 \mathrm{~W}$ peak power). With the CW signal beam in, the output at $1550 \mathrm{~nm}$ appeared in the form of spikes with a period of $200 \mu \mathrm{s}$ (red line in Figure 4c), which was synchronized with the repetition rate $(5 \mathrm{kHz})$ of the modulating pulses. Compared with many other all-optical modulators, such as silicon micro-ring modulators $^{39,40}$, the G/PVA nanofiber operated at a comparable modulating peak power of $\sim 3.2 \mathrm{~W}\left(\sim 16 \mathrm{~nJ}\right.$ pulse $\left.^{-1}\right)$ but has the advantages of a miniaturized size and great flexibility for integration. Considering that the response time of the graphene-based all-optical modulation is on 2-ps level ${ }^{19}$, the switching energy of the G/PVA nanofiber can go below $10 \mathrm{pJ}$.

\section{CONCLUSIONS}

To summarize, we have fabricated graphene-doped nanofibers with high optical quality. Due to the high optical confinement of waveguiding modes for enhanced light-matter interaction, the subwavelengthdiameter nanofiber exhibited saturable absorption behavior with a threshold down to $0.25 \mathrm{pJ}$ pulse ${ }^{-1}$ (peak power $\sim 1.3 \mathrm{~W}$ ). As a onedimensional structure, the G/PVA nanofiber has a much smaller overall size than many other graphene-incorporated two-dimensional structures (e.g., films or membranes) and can be used to fabricate nanophotonic devices with much more compact sizes, lower thresholds, and lower operation power. Additionally, nanofibers are compatible with and can be readily integrated into a mature platform of fiber optics without sacrificing their merits, such as low loss and polarization independent waveguiding. As a nanoscale nonlinear optical waveguide, the G/PVA nanofiber may open opportunities for diverse applications in future nanophotonic circuits and devices.

\section{ACKNOWLEDGEMENTS}

The authors thank Wei Li, Junhao He, Bigeng Chen, Huakang Yu, Weisong Yang, and Tao Chen for their help in nanofiber preparation and characterization. This work was supported by the National Natural Science Foundation of China (61475140) and the National Basic Research Program of China (2013CB328703 and 2012CB921400), Y. Ron. Shen was supported by the Director, Office of Science, Office of Basic Energy Sciences, Materials Sciences and Engineering Division, of the U.S. Department of Energy (DEAC03-76SF00098).

1 Novoselov KS, Geim AK, Morozov SV, Jiang D, Katsnelson MI et al. Two-dimensional gas of massless Dirac fermions in graphene. Nature 2005; 438: 197-200.

2 Nair RR, Blake P, Grigorenko AN, Novoselov KS, Booth TJ et al. Fine structure constant defines visual transparency of graphene. Science 2008; 320: 1308.

3 Bonaccorso F, Sun Z, Hasan T, Ferrari AC. Graphene photonics and optoelectronics. Nat Photonics 2010; 4: 611-622.

4 Wang F, Zhang YB, Tian CS, Girit C, Zettl A et al. Gate-variable optical transitions in graphene. Science 2008; 320: 206-209.

5 Li ZQ, Henriksen EA, Jiang Z, Hao Z, Martin MC et al. Dirac charge dynamics in graphene by infrared spectroscopy. Nat Phys 2008; 4: 532-535.

6 Bolotin KI, Sikes KJ, Jiang Z, Klima M, Fudenberg G et al. Ultrahigh electron mobility in suspended graphene. Solid State Commun 2008; 146: 351-355.

7 Dawlaty JM, Shivaraman S, Chandrashekhar M, Rana F, Spencer MG. Measurement of ultrafast carrier dynamics in epitaxial graphene. App/ Phys Lett 2008; 92: 042116042118.

8 Breusing M, Ropers C, Elsaesser T. Ultrafast carrier dynamics in graphite. Phys Rev Lett 2009; 102: 086809-086812.

9 Kumar S, Anija M, Kamaraju N, Vasu KS, Subrahmanyam KS et al. Femtosecond carrier dynamics and saturable absorption in graphene suspensions. Appl Phys Lett 2009; 95: 191911-191913.

10 Song YW, Jang SY, Han WS, Bae MK. Graphene mode-lockers for fiber lasers functioned with evanescent field interaction. Appl Phys Lett 2010; 96: 051122 051124.

11 Bao QL, Zhang H, Wang B, Ni ZH, Lim CHYX et al. Broadband graphene polarizer. Nat Photonics 2011; 5: 411-415.

$12 \mathrm{Kim} \mathrm{K}$, Choi JY, Kim T, Cho SH, Chung HJ. A role for graphene in silicon-based semiconductor devices. Nature 2011; 479: 338-344.

13 Liu M, Yin XB, Ulin-Avila E, Geng BS, Zentgraf T et al. A graphene-based broadband optical modulator. Nature 2011; 474: 64-67.

14 Bao QL, Loh KP. Graphene photonics, plasmonics, and broadband optoelectronic devices. ACS Nano 2012; 6: 3677-3694.

15 Furchi M, Urich A, Pospischil A, Lilley G, Unterrainer K et al. Microcavity-integrated graphene photodetector. Nano Lett 2012; 12: 2773-2777.

16 Koester SJ, Li M. High-speed waveguide-coupled graphene-on-graphene optical modulators. Appl Phys Lett 2012; 100: 171107-171110.

17 Liu M, Yin XB, Zhang X. Double-layer graphene optical modulator. Nano Lett 2012; 12: $1482-1485$.

18 Li H, Anugrah Y, Koester SJ, Li M. Optical absorption in graphene integrated on silicon waveguides. App/ Phys Lett 2012; 101: 111110-111114.

19 Li W, Chen BG, Meng C, Fang W, Xiao Y et al. Ultrafast all-optical graphene modulator Nano Lett 2014; 14: 955-959.

20 Tong LM, Lou JY, Mazur E. Single-mode guiding properties of subwavelengthdiameter silica and silicon wire waveguides. Opt Express 2004; 12: 1025-1035. 
21 Gu FX, Yu HK, Wang P, Yang ZY, Tong LM. Light-emitting polymer single nanofibers via waveguiding excitation. ACS Nano 2010; 4: 5332-5338.

22 Meng C, Xiao Y, Wang P, Zhang L, Liu YX et al. Quantum-dot-doped polymer nanofibers for optical sensing. Adv Mater 2011; 23: 3770-3774.

23 Wang P, Zhang L, Xia YN, Tong LM, Xu X et al. Polymer nanofibers embedded with aligned gold nanorods: a new platform for plasmonic studies and optical sensing. Nano Lett 2012; 12: 3145-3150.

24 Wang P, Wang YP, Tong LM. Functionalized polymer nanofibers: a versatile platform for manipulating light at the nanoscale. Light Sci App/2013; 2: e102, doi:10.1038/ Isa.2013.58.

25 Guo X, Ying YB, Tong LM. Photonic nanowires: from subwavelength waveguides to optical sensors. Acc Chem Res 2014; 47: 656-666.

26 Yang XG, Xu R, Bao DH, Li BJ. Gold nanorod-enhanced light emission in quantum-dotdoped polymer nanofibers. ACS App/ Mater Interfaces 2014; 6: 11846-11850.

27 Yan YL, Zhang C, Yao JN, Zhao YS. Recent advances in organic one-dimensional composite materials: design, construction, and photonic elements for information processing. Adv Mater 2013; 25: 3627-3638.

28 Garreau A, Duvail JL. Recent advances in optically active polymer-based nanowires and nanotubes. Adv Optic Mater 2014; 2: 1122-1140.

29 Coleman JN. Liquid exfoliation of defect-free graphene. Acc Chem Res 2013; 46 : $14-22$.

30 Lotya M, Hernandez Y, King PJ, Smith RJ, Nicolosi V et al. Liquid phase production of graphene by exfoliation of graphite in surfactant/water solutions. J Am Chem Soc 2009; 131: 3611-3620.

31 Bao QL, Zhang H, Yang JX, Wang S, Tong DY et al. Graphene-polymer nanofiber membrane for ultrafast photonics. Adv Funct Mater 2010; 20: 782-791.

32 Sun ZP, Hasan T, Torrisi F, Popa D, Privitera G et al. Graphene mode-locked ultrafast laser. ACS Nano 2010; 4: 803-810.
33 Ferrari AC, Meyer JC, Scardaci V, Casiraghi C, Lazzeri M et al. Raman spectrum of graphene and graphene layers. Phys Rev Lett 2006; 97: 187401-187404.

34 Fasano V, Polini A, Morello G, Moffa M, Camposeo A et al. Bright light emission and waveguiding in conjugated polymer nanofibers electrospun from organic salt added solutions. Macromolecules 2013; 46: 5935-5942.

35 O'Carroll D, Lieberwirth I, Redmond G. Melt-processed polyfluorene nanowires as active waveguides. Small 2007; 3: 1178-1183.

36 Bao QL, Zhang H, Wang Y, Ni ZH, Yan YL et al. Atomic-layer graphene as a saturable absorber for ultrafast pulsed lasers. Adv Funct Mater 2009; 19: 3077 3083.

37 Vasko FT. Saturation of interband absorption in graphene. Phys Rev B 2010; 82 : $245422-245427$.

38 Popa D, Sun Z, Torrisi F, Hasan T, Wang F et al. Sub 200 fs pulse generation from a graphene mode-locked fiber laser. Appl Phys Lett 2010; 97: 203106-203108.

39 Almeida VR, Barrios CA, Panepucci RR, Lipson M. All-optical control of light on a silicon chip. Nature 2004; 431: 1081-1084.

40 Preston K, Dong P, Schmidt B, Lipson M. High-speed all-optical modulation using polycrystalline silicon microring resonators. Appl Phys Lett 2008; 92: 151104151106.

(c) (i) (2) This license allows readers to copy, distribute and transmit the Contribution transform or build upon the Contribution as long as the resulting work is then distributed under this or a similar license. Readers are not permitted to use the Contribution for commercial purposes. Please read the full license for further details at - http:// creativecommons.org/licenses/by-nc-sa/4.0/

Supplementary information for this article can be found on the Light: Science \& Applications' website (http://www.nature.com/lsa/). 